\title{
ON A CLASS OF FUNCTIONS DEFINED BY PARTIAL DIFFERENTIAL EQUATIONS
}

\author{
BY \\ LIPMAN BERS AND ABE GELBART(1)
}

1. Introduction. Various problems in mechanics of continua lead to a system of differential equations of the form

$$
\begin{aligned}
& \sigma_{1}(x) u_{x}=\tau_{1}(y) v_{y}, \\
& \sigma_{2}(x) u_{y}=-\tau_{2}(y) v_{x},
\end{aligned}
$$

where $u=u(x, y), v=v(x, y)$, and subscripts denote partial differentiation( $\left.{ }^{2}\right)$.

The similarity between this system and the Cauchy-Riemann equations suggests the study of complex-valued functions

$$
f(x+i y)=u(x, y)+i v(x, y),
$$

whose real and imaginary parts are connected by the equations (1.1). Many concepts and results in the theory of analytic functions of a complex variable can be extended to functions satisfying the system (1.1), notably the concept of differentiation, integration, powers, power series, the theorem of Cauchy and Morera and the fundamental theorem of algebra $\left({ }^{3}\right)$.

In what follows we make the following assumptions concerning the coefficients $\sigma_{i}, \tau_{j}$.

Hypothesis $\alpha . \sigma_{i}$ and $\tau_{j}$ are defined for all values of their respective real variables.

This assumption is made mainly for the sake of convenience. ables.

Hypothesis $\beta . \sigma_{i}$ and $\tau_{j}$ are analytic functions of their respective real vari-

Presented to the Society, October 30, 1943; received by the editors February 4, 1944.

(1) The results of this paper were obtained while the authors participated in the program of Advanced Instruction and Research in Mechanics at Brown University, Summer, 1942. The authors wish to express their appreciation to Professor Prager for the many profitable discussions they had with him and for his constant encouragement.

(2) Cf. [4] where the applied aspect of the same problem is treated. Numbers in brackets refer to the references cited at the end of the paper.

$\left({ }^{3}\right)$ The idea of defining classes of functions similar to the class of analytic functions by means of systems of partial differential equations has been announced by Picard in 1891 (cf. $[5,6])$. A. Weinstein kindly drew our attention to the fact that for the case $\sigma_{1}=\sigma_{2}=1$, $\tau_{1}=\tau_{2}=1 / y$ Beltrami defined a process identical to our "differentiation" and "integration" (cf. $[1,2]$ ). There also exist other methods of applying function-theoretic results to the study of partial differential equations, notably S. Bergman's method of integral operators (cf. [3] and the papers quoted therein). 
For many purposes this hypothesis can be replaced by

Hypothesis $\beta^{\prime}$. $\sigma_{i}$ and $\tau_{j}$ are continuously differentiable.

HyPOTHESIS $\gamma, \sigma_{i}$ and $\tau_{j}$ are positive.

This assumption is essential. It insures that the second order equations which are obtained from (1.1) by eliminating either $v$ or $u$ :

$$
\begin{array}{r}
\left(\left(\sigma_{1} / \tau_{1}\right) u_{x}\right)_{x}+\left(\left(\sigma_{2} / \tau_{2}\right) u_{y}\right)_{y}=0, \\
\left(\left(\tau_{2} / \sigma_{2}\right) v_{x}\right)_{x}+\left(\left(\tau_{1} / \sigma_{1}\right) v_{y}\right)_{y}=0,
\end{array}
$$

be of elliptic type. Hypothesis $\gamma$ can be replaced by

HyPOTHESIS $\gamma^{\prime}, \sigma_{1} \sigma_{2} \tau_{1} \tau_{2}>0$.

Some of our results hold also for the hyperbolic case, $\sigma_{1} \sigma_{2} \tau_{1} \tau_{2}<0$ (cf. §11). At one point we shall also assume

Hy pothesis $\delta$. The limits $\sigma_{i}(+\infty), \sigma_{j}(-\infty), \tau_{i}(+\infty), \tau_{j}(-\infty), i, j=1,2$, exist and are not equal to $0, \infty$.

Together with (1.1) it is desirable to consider the associated system

$$
\begin{aligned}
& u_{x} / \sigma_{2}(x)=\tau_{1}(y) v_{y}, \\
& u_{y} / \sigma_{1}(x)=-\tau_{2}(y) v_{x} .
\end{aligned}
$$

The coefficient matrices of (1.1) and (1.4) will be denoted by $\Sigma$ and $\Sigma^{\prime}$ respectively. Thus

$$
\Sigma=\left\|\begin{array}{cc}
\sigma_{1} & \tau_{1} \\
\sigma_{2} & \tau_{2}
\end{array}\right\|, \quad \Sigma^{\prime}=\left\|\begin{array}{cc}
1 / \sigma_{2} & \tau_{1} \\
1 / \sigma_{1} & \tau_{2}
\end{array}\right\|,
$$

and

$$
\Sigma^{\prime \prime} \equiv \Sigma
$$

We shall also use the matrix

$$
\Sigma(\lambda)=\left\|\begin{array}{cc}
\sigma_{1}^{\lambda} & \tau_{1}^{\lambda} \\
{\sigma_{2}{ }^{\lambda}}^{\lambda}{ }^{\lambda} \tau_{2}{ }^{\lambda}
\end{array}\right\|,
$$

$\lambda$ being a real parameter. Plainly

$$
\Sigma(1)=\Sigma, \quad \Sigma(\lambda)^{\prime}=\Sigma^{\prime}(\lambda)
$$

If hypothesis $\delta$ is satisfied as well as the hypotheses $\alpha, \beta$ (or $\beta^{\prime}$ ), and $\gamma$, we call $\Sigma$ a normal matrix. If $\Sigma$ is normal, so are $\Sigma^{\prime}$ and $\Sigma(\lambda)$. 
We shall often use the non-decreasing function $m_{\Sigma}(A)$, defined by

$$
m_{\Sigma}(A)=\max _{i=1,2} \max _{j= \pm 1} \max _{|i| \leqq \Lambda}\left\{\sigma_{i}(t)^{j}, \tau_{i}(t)^{j}\right\} \text {. }
$$

Note that

$$
m_{\Sigma}(A)=m_{\Sigma}(A)
$$

and

$$
m_{\Sigma(\lambda)}(A) \leqq m_{\Sigma}(A)
$$$$
\text { for } 0 \leqq \lambda \leqq 1 \text {. }
$$

If $m_{\Sigma} \equiv 1$, then

$$
\Sigma=\left\|\begin{array}{ll}
1 & 1 \\
1 & 1
\end{array}\right\|
$$

and (1.1) coincides with the Cauchy-Riemann equations.

2. Differentiation. $\Sigma$-monogenic functions. Let $f=u+i v$ be an arbitrary function defined in some neighborhood of a (fixed) point $z=x+i y$. We define its $\Sigma$-difference quotient at $z$ as

$$
\begin{aligned}
\Delta_{\Sigma} f(z) / \Delta_{\Sigma} z= & \Re\left\{(\Delta z)^{-1}\left[\sigma_{1}(x) \Delta_{1} u+i \tau_{1}(y) \Delta_{2} v\right]\right\} \\
& +i \Im\left\{(\Delta z)^{-1}\left[\Delta_{2} u / \tau_{2}(y)+i \Delta_{1} v / \sigma_{2}(x)\right]\right\}
\end{aligned}
$$

where $\Delta z$ is an arbitrary complex variable of sufficiently small modulus,

$$
\begin{aligned}
& \Delta_{1} u=2^{-1}[u(z+\Delta z)+u(z+\overline{\Delta z})-2 u(z)], \\
& \Delta_{2} u=2^{-1}[u(z+\Delta z)+u(z-\overline{\Delta z})-2 u(z)],
\end{aligned}
$$

and $\Delta_{1} v$ and $\Delta_{2} v$ are defined similarly. We define the $\Sigma$-derivative of $f(z)$ (at z) as

$$
d_{\Sigma} f(z) / d_{\Sigma} z=f^{\prime}(z)=\lim _{\Delta z \rightarrow 0}\left(\Delta_{\Sigma} f(z) / \Delta_{\Sigma} z\right)
$$

provided the limit exists and is finite.

ThEOREM 2.1. If $f=u+i v$ possesses $a \mathbf{\Sigma}$-derivative at $z$, then $u$ and $v$ possess partial derivatives at this point and

$$
f^{\prime}(z)=\sigma_{1} u_{x}+\left(i / \sigma_{2}\right) v_{x}=\tau_{1} v_{y}-\left(i / \tau_{2}\right) u_{y}
$$

so that $u$ and $v$ satisfy (1.1). Conversely, if $u$ and $v$ possess continuous partiai derivatives at $z$ that satisfy (1.1), then $f$ possesses a $\Sigma$-derivative at this point.

Proof. The first assertion follows immediately from the definition of the $\Sigma$-derivative (by taking $\Delta z$ first as a real and then as a purely imaginary quantity). On the other hand, assuming the existence of continuous partial derivatives and setting

$$
\Delta z=r e^{i \theta},
$$

we have by the mean value theorem 


$$
\begin{aligned}
2 \Delta_{\Sigma} f(z) / \Delta_{\Sigma} z= & \left\{\sigma_{1}(x)\left[u_{x}\left(z+r_{1} e^{i \theta}\right)+u_{x}\left(z+r_{2} e^{-i \theta}\right)\right] \cos ^{2} \theta\right. \\
& \left.+\tau_{1}(y)\left[v_{y}\left(z+r_{4} e^{i \theta}\right)+v_{y}\left(z-r_{6} e^{-i \theta}\right)\right] \sin ^{2} \theta\right\} \\
& +\left\{\sigma_{1}(x)\left[u_{y}\left(z+r_{1} e^{i \theta}\right)-u_{y}\left(z+r_{2} e^{-i \theta}\right)\right]\right. \\
& \left.+\tau_{1}(y)\left[v_{x}\left(z+r_{4} e^{i \theta}\right)-v_{x}\left(z-r_{6} e^{-i \theta}\right)\right]\right\} \sin \theta \cos \theta \\
& +i\left\{\left(\tau_{2}(y)\right)^{-1}\left[-u_{y}\left(z+r_{1} e^{i \theta}\right)-u_{y}\left(z-r_{3} e^{-i \theta}\right)\right] \sin ^{2} \theta\right. \\
& \left.+\left(\sigma_{2}(x)\right)^{-1}\left[v_{x}\left(z+r_{4} e^{i \theta}\right)+v_{x}\left(z+r_{5} e^{-i \theta}\right)\right] \cos ^{2} \theta\right\} \\
& +i\left\{\left(\tau_{2}(y)\right)^{-1}\left[-u_{x}\left(z+r_{1} e^{i \theta}\right)+u_{x}\left(z-r_{3} e^{-i \theta}\right)\right]\right. \\
& \left.+\left(\sigma_{2}(x)\right)^{-1}\left[v_{y}\left(z+r_{4} e^{i \theta}\right)-v_{y}\left(z+r_{5} e^{-i \theta}\right)\right]\right\} \sin \theta \cos \theta \\
= & \{1\}+\{2\} \sin \theta \cos \theta+i\{3\}+i\{4\} \sin \theta \cos \theta
\end{aligned}
$$

where

$$
0 \leqq r_{i} \leqq r, \quad i=1,2, \cdots, 6 .
$$

When $r \rightarrow 0$, then, independently of $\theta,\{2\} \rightarrow 0,\{4\} \rightarrow 0$ (since the partial derivatives are continuous), and $\{1\}$ and $\{3\}$ possess limits by virtue of (1.1).

A function $f(z)$ possessing a $\Sigma$-derivative at $z$ and at all points of some neighborhood of $z$ shall be called $\Sigma$-monogenic at $z$. If $m_{\Sigma}(A) \equiv 1$, then

$$
f^{\prime}(z)=f^{\prime}(z)
$$

and a $\Sigma$-monogenic function is analytic.

Some immediate consequences of Theorem 2.1 and of our definition follow.

$A \Sigma$-monogenic function is continuous.

$A$ constant is $\Sigma$-monogenic; and any function with an identically vanishing $\Sigma$-derivative is constant.

If $f$ and $g$ are $\Sigma$-monogenic and $\alpha$ and $\beta$ are real constants, then $\alpha f+\beta g$ is $\Sigma$-monogenic and

$$
(\alpha f+\beta g)^{\prime}=\alpha f^{\prime}+\beta g^{\prime} .
$$

Note that if $a$ is a complex constant and if $f$ is a $\Sigma$-monogenic function, $a f$ is not in general $\Sigma$-monogenic. Neither are $f(z) g(z), f[g(z)]$ ( $f$ and $g$ being $\Sigma$ monogenic) nor the function $z=f^{-1}(w)(w=f(z))$.

REMARK. In the symmetric case $\left({ }^{4}\right), \sigma_{1}=\sigma_{2}=\sigma, \tau_{1}=\tau_{2}=\tau$, the definition (2.1) may be replaced by the simpler one:

$$
\begin{aligned}
\Delta_{\Sigma} f / \Delta_{\Sigma} z= & \Re\left\{(\Delta z)^{-1}[\sigma(x) \Delta u+i \tau(y) \Delta v]\right\} \\
& +i \Im\left\{(\Delta z)^{-1}[\Delta u / \tau(y)+i \Delta v / \sigma(x)]\right\},
\end{aligned}
$$

where

$$
\Delta u=u(z+\Delta z)-u(z)
$$

and $\Delta v$ is defined similarly.

( ${ }^{4}$ By introducing new independent variables $\xi=\phi(x), \eta=\psi(y)$ the system (1.1) can be put into a simpler form; for instance, into a form with $\sigma_{1}=\sigma_{2}, \tau_{1}=\tau_{2}$, or a form with $\sigma_{1}=\tau_{1}=1$. 
3. Integration. We define the $\Sigma$-integral of a continuous function $f(z)$ over a rectifiable curve $C$ by

$$
\int_{C} f(z) d_{\Sigma} z=\int_{C}\left(\sigma_{2} u d x-\tau_{2} v d y\right)+i \int_{C}\left(\left(v / \sigma_{1}\right) d x+\left(u / \tau_{1}\right) d y\right) .
$$

Clearly

$$
\int_{C}(\alpha f+\beta g) d_{\Sigma z}=\alpha \int_{C} f d_{\Sigma z}+\beta \int_{C} g d_{\Sigma z}
$$

$\alpha$ and $\beta$ being real constants, and (over $C$ )

$$
\int_{a}^{b} f d_{\Sigma} z+\int_{b}^{c} f d_{\Sigma} z=\int_{a}^{c} f d_{\Sigma} z
$$

$a, b, c$ being points on the curve. A $\Sigma$-integral over $C$ can be approximated by a $\Sigma$-integral over a polygon.

Since (3.1) can be written in the form

where

$$
\int_{C} f d_{\Sigma z}=\Re \int_{C} g d z+i \Im \int_{C} h d z
$$

$$
g=\sigma_{2} u+i \tau_{2} v, \quad h=u / \tau_{1}+i v / \sigma_{1},
$$

we have the crude but helpful inequality

$$
\left|\int_{C} f d_{\Sigma} z\right| \leqq 2 m_{\Sigma}(A) \max _{C}|f| l,
$$

where $l$ is the length of $C, A$ a constant such that for all points of $C,|x| \leqq A$, $|y| \leqq A$, and $m_{\Sigma}$ the function defined by (1.8). Plainly (3.2) can be replaced by

$$
\left|\int_{C} f d_{\Sigma z}\right| \leqq 2 m_{\Sigma}(A) \int_{C}|f| d s,
$$

$d s$ being the arc length element on $C$.

THEOREM 3.1. If $f(z)$ is $\Sigma$-monogenic in a closed simply connected domain $D$ bounded by a rectifiable curve $C$, then

$$
\int_{C} f(z) d_{\Sigma} z=0 .
$$

Proof. The theorem can be proved by a reasoning similar to that used in Goursat's proof of Cauchy's theorem. A much shorter proof can be given, if we assume an extension of a theorem of Menshoff and Looman, which was suggested by Miss Lorenz and announced without proof by Saks $\left.{ }^{\mathfrak{5}}\right)$.

(5) [7 pp. 242-243]. 
Consider the real part of (3.4),

$$
\int_{C} \sigma_{2} u d x-\tau_{2} v d y
$$

We know that the partial derivatives $u_{x}, u_{y}, v_{x}$ exist. It follows from assumption $\beta$ that $\left(\sigma_{2} u\right)_{x},\left(\sigma_{2} u\right)_{y},\left(\tau_{2} v\right)_{x},\left(\tau_{2} v\right)_{y}$ also exist. By $(1.1),\left(\sigma_{2} u\right)_{y}=\left(-\tau_{2} v\right)_{x}$. This implies that (3.5) vanishes, by virtue of the theorem mentioned above. The same holds for the imaginary part of (3.4). From this proof it follows that

Theorem 3.1 also holds if assumption $\beta$ is replaced by $\beta^{\prime}$.

Now let $f$ be a $\Sigma$-monogenic function and

We have

$$
F(z)=\int_{a}^{z} f d_{\Sigma z}=U+i V .
$$

$$
U_{x}=\sigma_{2} u, \quad U_{y}=-\tau_{2} v, \quad V_{x}=v / \sigma_{1}, \quad V_{y}=u / \tau_{1},
$$

that is,

$$
\begin{aligned}
& U_{x} / \sigma_{2}=\tau_{1} V_{y}, \\
& U_{y} / \sigma_{1}=-\tau_{2} V_{x} .
\end{aligned}
$$

Since the partial derivatives (3.6) are continuous we have

THEOREM 3.2. The $\Sigma$-integral of a $\Sigma$-monogenic function is $\Sigma^{\prime}$-monogenic.

Next set

$$
G(z)=\int_{a}^{z} F d_{\Sigma^{\prime} z}=\phi+i \psi .
$$

By Theorem 3.2 and (1.6), $G$ is $\Sigma$-monogenic. Furthermore, $\phi$ and $\psi$ possess continuous partial derivatives of the second order. Since they are connected by equations (1.1), $\phi$ satisfies (1.3a) and $\psi(1.3 \mathrm{~b})$. These equations are of elliptic type and have analytic coefficients. Hence $\phi$ and $\psi$ are analytic. Since

$$
u=\left(\tau_{1} / \sigma_{2}\right) \psi_{x y}, \quad v=-\left(\sigma_{1} / \tau_{2}\right) \phi_{x y},
$$

we have

THEOREM 3.3. The real and the imaginary parts of a $\Sigma$-monogenic function are analytic functions of $x$ and $y$.

This implies

THEOREM 3.4. The $\Sigma$-derivative of a $\Sigma$-monogenic function is $\Sigma^{\prime}$-monogenic. In fact, setting

$$
f^{\prime}=u^{\prime}+i v^{\prime}
$$


we have

$$
\begin{aligned}
& u_{x}^{\prime} / \sigma_{2}=\left(\tau_{1} v_{y}\right)_{x} / \sigma_{2}=\left(\tau_{1} / \sigma_{2}\right) v_{x y}=\tau_{1}\left(v_{x} / \sigma_{2}\right)_{y}=\tau_{1} v_{y}^{\prime}, \\
& u_{y}^{\prime} / \sigma_{1}=\left(\sigma_{1} u_{x}\right)_{y} / \sigma_{1}=u_{x y}=\tau_{2}\left(u_{y} / \tau_{2}\right)_{x}=-\tau_{2} v_{x}^{\prime} .
\end{aligned}
$$

Thus unlimited $\Sigma$ and $\Sigma^{\prime}$-differentiations of a $\Sigma$-monogenic function, $f$, are possible.

THEOREM 3.5. $\Sigma$-differentiation and $\Sigma^{\prime}$-integration as well as $\Sigma^{\prime}$-differentition and $\Sigma$-integration are inverse processes, that is,

$$
\frac{d}{d_{\Sigma^{\prime} z}} \int_{a}^{z} f(\zeta) d_{\Sigma} \zeta=f(z), \quad \int_{a}^{b} f^{\prime}(\zeta) d_{\Sigma^{\prime}} \zeta=f(b)-f(a)
$$

where $f$ is a $\Sigma$-monogenic function.

This follows simply by comparing formula (2.3) and (3.1).

The preceding considerations contain also the proof of

THEOREM 3.6. If $f(z)$ is continuous in a simply connected domain $D$ and if for every simple closed rectifiable curve $C$ in $D, \int c f d_{\Sigma} z=0, f$ is $\Sigma$-monogenic in $D\left({ }^{6}\right)$.

\section{This implies}

THEOREM 3.7. The limit of a uniformly convergent sequence of $\Sigma$-monogenic functions is $\Sigma$-monogenic.

For such a sequence can be $\Sigma$-integrated term by term.

REMARK. Let us replace hypothesis $\beta$ by $\beta^{\prime}$. Then Theorem 3.2 is still true, Theorem 3.3 obviously false, Theorem 3.4 true only if $u$ and $v$ possess continuous partial derivatives of the second order and Theorem 3.5 is true. Unlimited $\Sigma$ or $\Sigma^{\prime}$-differentiation is, in general, impossible.

4. Formal powers. Since a (complex) constant is $\Sigma$-monogenic, we can construct $\Sigma$-monogenic functions by iterated $\Sigma$ - and $\Sigma^{\prime}$-integrations of a constant. We define ( $a$ and $z_{0}$ being constants) $\left.{ }^{7}\right)$

$$
\begin{aligned}
& a \cdot Z^{(0)}\left(z_{0} ; z\right)=a \cdot \tilde{Z}^{(0)}\left(z_{0} ; z\right)=a, \\
& a \cdot Z^{(n)}\left(z_{0} ; z\right)=n \int_{z_{0}}^{z}\left(a \cdot \tilde{Z}^{(n-1)}\left(z_{0} ; z\right)\right) d_{\Sigma} z, \\
& a \cdot \tilde{Z}^{(n)}\left(z_{0} ; z\right)=n \int_{z_{0}}^{z}\left(a \cdot Z^{(n-1)}\left(z_{0} ; z\right)\right) d_{\Sigma} z .
\end{aligned}
$$

By definition $a \cdot Z^{(n)}$ is $\Sigma$-monogenic and $a \cdot \tilde{Z}^{(n)} \Sigma^{\prime}$-monogenic. Furthermore (for $n \geqq 1$ )

(6) Analogue of Morera's theorem.

(7) In the following formulas $a \cdot Z$ is one symbol. 


$$
\begin{gathered}
a \cdot Z^{(n)}\left(z_{0} ; z_{0}\right)=a \cdot \tilde{Z}^{(n)}\left(z_{0} ; z_{0}\right)=0 \\
\left\{\begin{array}{l}
\frac{d_{\Sigma}}{d_{\Sigma^{z}}}\left(a \cdot Z^{(n)}\left(z_{0} ; z\right)\right)=n a \cdot \tilde{Z}^{(n-1)}\left(z_{0} ; z\right) \\
\frac{d_{\Sigma^{\prime}}}{d_{\Sigma^{\prime}} z}\left(a \cdot \tilde{Z}^{(n)}\left(z_{0} ; z\right)\right)=n a \cdot Z^{(n-1)}\left(z_{0} ; z\right) .
\end{array}\right.
\end{gathered}
$$

For the sake of brevity we set $1 \cdot Z^{(n)}\left(z_{0} ; z\right)=Z^{(n)}\left(z_{0} ; z\right), 1 \cdot \tilde{Z}^{(n)}\left(z_{0} ; z\right)=\tilde{Z}^{(n)}\left(z_{0} ; z\right)$, $a \cdot Z^{(n)}(0 ; z)=a \cdot Z^{(n)}(z), a \cdot \tilde{Z}^{(n)}(0 ; z)=a \cdot \tilde{Z}^{(n)}(z)$. Obviously, for real $\alpha$ and $\beta$,

$$
(\alpha+i \beta) \cdot Z^{(n)}\left(z_{0} ; z\right)=\alpha Z^{(n)}\left(z_{0} ; z\right)+\beta i \cdot Z^{(n)}\left(z_{0} ; z\right)
$$

and similarly for $\tilde{Z}^{(n)}\left(z_{0} ; z\right)$.

We call $a \cdot Z^{(n)}$ the "formal product" of $a$ with the "formal power" $Z^{(n)}\left(z_{0} ; z\right)$ (cf. §8). If $m_{\Sigma} \equiv 1$, then $a \cdot Z^{(n)}\left(z_{0} ; z\right)=a\left(z-z_{0}\right)^{n}$.

The real and imaginary parts of the formal powers $a \cdot Z^{(n)}\left(z_{0} ; z\right)$ furnish an unlimited number of particular solutions of the equations (1.3a) and (1.3b). For the handling of these formal powers it is convenient to use the following formulas, formally equivalent to the binomial formula for $\left(z-z_{0}\right)^{n}\left(^{8}\right)$. We first introduce the following (real) functions:

$$
\begin{aligned}
& X^{(0)}\left(x_{0} ; x\right)=X^{*(0)}\left(x_{0} ; x\right)=Y^{(0)}\left(y_{0} ; y\right)=Y^{*(0)}\left(y_{0} ; y\right)=1, \\
& X^{(n)}\left(x_{0} ; x\right)=\left\{\begin{array}{l}
n \int_{x_{0}}^{x} \frac{1}{\sigma_{1}(\xi)} X^{(n-1)}\left(x_{0} ; \xi\right) d \xi \text { if } n \text { is odd, } \\
n \int_{x_{0}}^{x} \sigma_{2}(\xi) X^{(n-1)}\left(x_{0} ; \xi\right) d \xi \text { if } n \text { is even, }
\end{array}\right. \\
& X^{*(n)}\left(x_{0} ; x\right)=\left\{\begin{array}{l}
n \int_{x_{0}}^{x} \sigma_{2}(\xi) X^{*(n-1)}\left(x_{0} ; \xi\right) d \xi \text { if } n \text { is odd, } \\
n \int_{x_{0}}^{x} \frac{1}{\sigma_{1}(\xi)} X^{*(n-1)}\left(x_{0} ; \xi\right) d \xi \text { if } n \text { is even, }
\end{array}\right. \\
& Y^{(n)}\left(y_{0} ; y\right)= \begin{cases}n \int_{y_{0}}^{y} \frac{1}{\tau_{1}(\eta)} Y^{(n-1)}\left(y_{0} ; \eta\right) d \eta & \text { if } n \text { is odd, } \\
n \int_{y_{0}}^{y} \tau_{2}(\eta) Y^{(n-1)}\left(y_{0} ; \eta\right) d \eta & \text { if } n \text { is even, }\end{cases} \\
& Y^{*(n)}\left(y_{0} ; y\right)=\left\{\begin{array}{l}
n \int_{y_{0}}^{y} \tau_{2}(\eta) Y^{*(n-1)}\left(y_{0} ; \eta\right) d \eta \text { if } n \text { is odd, } \\
n \int_{y_{0}}^{y} \frac{1}{\tau_{1}(\eta)} Y^{*(n-1)}\left(y_{0} ; \eta\right) d \eta \text { if } n \text { is even. }
\end{array}\right.
\end{aligned}
$$

(8) Formula (9.2) is another analogue of the binomial formula. 
In other words:

$$
\begin{aligned}
& X^{(n)}\left(x_{0} ; x\right)=n ! \int_{x_{0}}^{x} \frac{1}{\sigma_{1}} \int_{x_{0}} \sigma_{2} \cdots \int_{x_{0}} \sigma_{2} \int_{x_{0}} \frac{d x^{n}}{\sigma_{1}}(n \text { integrals, } n \text { odd }), \\
& X^{(n)}\left(x_{0} ; x\right)=n ! \int_{x_{0}}^{x} \sigma_{2} \int_{x_{0}} \frac{1}{\sigma_{1}} \cdots \int_{x_{0}} \sigma_{2} \int_{x_{0}} \frac{d x^{n}}{\sigma_{1}}(n \text { integrals, } n \text { even }),
\end{aligned}
$$

and similarly for $X^{*(n)}, Y^{(n)}, Y^{*(n)}$. If $x_{0}=0$ (or $y_{0}=0$ ), we merely omit it.

Now we have (for $z_{0}=x_{0}+i y_{0}$ )

$$
\begin{aligned}
& Z^{(n)}\left(z_{0} ; z\right)=\sum_{\nu=0}^{n} C_{n, \nu} i^{n-\nu} X^{(\nu)}\left(x_{0} ; x\right) Y^{(n-\nu)}\left(y_{0} ; y\right), \\
& \tilde{Z}^{(n)}\left(z_{0} ; z\right)=\sum_{\nu=0}^{n} C_{n, \nu} i^{n-\nu} X^{*(\nu)}\left(x_{0} ; x\right) Y^{(n-\nu)}\left(y_{0} ; y\right) \text {, } \\
& \text { (4.6) } i \cdot Z^{(n)}\left(z_{0} ; z\right)=\left\{\begin{array}{l}
i \sum_{\nu=0}^{n} C_{n, \nu} i^{n-\nu} X^{*(\nu)}\left(x_{0} ; x\right) Y^{*(n-\nu)}\left(y_{0} ; y\right) \text { if } n \text { is odd, } \\
i \sum_{\nu=0}^{n} C_{n, \nu} i^{n-\nu} X^{(\nu)}\left(x_{0} ; x\right) Y^{*(n-\nu)}\left(y_{0} ; y\right) \text { if } n \text { is even, }
\end{array}\right. \\
& i \cdot \tilde{Z}^{(n)}\left(z_{0} ; z\right)=\left\{\begin{array}{l}
i \sum_{\nu=0}^{n} C_{n, \nu} i^{n-\nu} X^{(\nu)}\left(x_{0} ; x\right) Y^{*(n-\nu)}\left(y_{0} ; y\right) \text { if } n \text { is odd, } \\
i \sum_{\nu=0}^{n} C_{n, \nu} i^{n-\nu} X^{*(\nu)}\left(x_{0} ; x\right) Y^{*(n-\nu)}\left(y_{0} ; y\right) \text { if } n \text { is even. }
\end{array}\right.
\end{aligned}
$$

These formulae can be checked easily by calculating the first few formal powers and then proved by induction.

In this section we did not use hypothesis $\beta$ but only $\beta^{\prime}$. Even a weaker hypothesis (continuity or integrability of the $\sigma_{i}, \tau_{j}$ ) would be sufficient.

5. Inequalities for formal powers. In this section we shall derive some auxiliary inequalities to be used later. These inequalities show that for very small and (under certain conditions) for very large values of $\left|z-z_{0}\right|$, $a \cdot Z^{(n)}\left(z_{0} ; z\right)$ behaves like an ordinary power.

First we note that by (1.8) and (4.5)

$$
m_{\Sigma}^{-n}\left(\left|x_{0}\right|+|x|\right) \leqq \frac{X^{(n)}\left(x_{0} ; x\right)}{\left(x-x_{0}\right)^{n}} \leqq m_{\Sigma}^{n}\left(\left|x_{0}\right|+|x|\right) .
$$

Similar inequalities are also true for $X^{*(n)}, Y^{(n)}, Y^{*(n)}$.

This implies

LEMMA 5.1. We have

$$
\left|a \cdot Z^{(n)}\left(z_{0} ; z\right)\right| \leqq 2^{1 / 2}|a|\left\{2^{1 / 2} m_{\Sigma}\left(\left|z_{0}\right|+|z|\right)\left|z-z_{0}\right|\right\}^{n} .
$$


For, setting $a=\alpha+i \beta$, we have by (5.1) and (4.6)

$$
\left|a \cdot Z^{(n)}\left(z_{0} ; z\right)\right|^{2} \leqq 2|a|^{2} m_{\Sigma}^{2 n}\left(\left|z_{0}\right|+|z|\right)\left(\left|x-x_{0}\right|+\left|y-y_{0}\right|\right)^{2 n} \text {. }
$$

We have also the somewhat deeper

LEMMA 5.2. Given two constants $a \neq 0$ and $z_{0}$, and $a$ positive integer $n$, there exist $a$ positive $\delta$ and $a$ positive $\epsilon$ (both depending upon $\Sigma$ ) such that

$$
\left|a \cdot Z^{(n)}\left(z_{0} ; z\right)\right|>\delta\left|z-z_{0}\right|^{n} \quad \text { for }\left|z-z_{0}\right| \leqq \epsilon .
$$

Proof. Without loss of generality we may assume $z_{0}=0$. We consider first two special cases:

(I) $\sigma_{1}(0)=\sigma_{2}(0)=\tau_{1}(0)=\tau_{2}(0)=1$,

(II) $\sigma_{1}(0)=\sigma_{2}(0)=\sigma_{0} \neq \tau_{0}=\tau_{1}(0)=\tau_{2}(0)$.

In case (I) we choose corresponding to any positive $\epsilon_{1}<1$ a positive $\epsilon_{2}=\epsilon_{2}\left(\epsilon_{1}\right)<\epsilon_{1}$ such that $\max \left\{\left|1-\sigma_{i}^{k}(t)\right|,\left|1-\tau_{i}^{k}(t)\right|\right\}<\epsilon_{1}$, for $|t| \leqq \epsilon_{2}, i=1,2$; $k= \pm 1$. Then $m_{\Sigma}\left(\epsilon_{2}\right)<1+\epsilon_{1}$ and

$$
\left|X^{(n)}(x)-x^{n}\right|<\epsilon_{1} c_{n}|x|^{n}, \quad|x| \leqq \epsilon_{2},
$$

where

$$
c_{1}=1 \text { and } c_{n+1}=1+2 c_{n} .
$$

For, assuming that $|x| \leqq \epsilon_{2}$,

$$
|X(x)-x|=\left|\int_{0}^{x}\left(1-1 / \sigma_{1}\right) d x\right|<\epsilon_{1}|x| ;
$$

and if (5.4) holds for $n-1$ we have (say for an even $n$ )

$$
\begin{aligned}
\left|X^{(n)}(x)-x^{n}\right| & =\left|\int_{0}^{x} n\left[\sigma_{2} X^{n-1}-x^{n-1}\right] d x\right| \\
& \leqq\left|n \int_{0}^{x} \sigma_{2}\left(X^{(n-1)}-x^{n-1}\right) d x\right|+\left|n \int_{0}^{x}\left(\sigma_{2}-1\right) x^{n-1} d x\right| \\
& <\epsilon_{1}\left(1+\epsilon_{1}\right) c_{n-1}|x|^{n}+\epsilon_{1}|x|^{n}<\epsilon_{1}\left(1+2 c_{n-1}\right)|x|^{n} .
\end{aligned}
$$

Similar inequalities hold for $X^{*(n)}, Y^{(n)}, Y^{*(n)}$.

Now

$$
\begin{aligned}
\left|Z^{(n)}(z)-z^{n}\right|= & \left|\sum_{\nu=0}^{n} C_{n, \nu}\left[i^{n-\nu} X^{(\nu)}(x) Y^{(n-\nu)}(y)-x^{\nu} y^{n-\nu}\right]\right| \\
\leqq & \sum_{\nu=0}^{n} C_{n, \nu}\left|X^{(\nu)}(x)-x^{\nu}\right|\left|Y^{(n-\nu)}(y)\right| \\
& +\sum_{\nu=0}^{n} C_{n, \nu}|x| \nu\left|Y^{n-\nu}(y)-y^{n-\nu}\right|=S_{1}+S_{2} .
\end{aligned}
$$


By (5.1) and (5.4) we have for $|z| \leqq \epsilon_{2}$

$$
\begin{aligned}
S_{1} & <c_{n} \epsilon_{1} \sum_{\nu=0}^{n} C_{n, \nu}|x|^{\nu}\left[m_{\Sigma}\left(\epsilon_{2}\right)|y|\right]^{n-\nu} \\
& <c_{n} \epsilon_{1}\left[|x|+\left(1+\epsilon_{1}\right)|y|\right]^{n}<2^{2 n} c_{n} \epsilon_{1}|z|^{n} .
\end{aligned}
$$

Similarly

$$
S_{2}<2{ }^{2 n} c_{n} \epsilon_{1}|z|^{n}
$$

so that

$$
\left|Z^{(n)}(z)-z^{n}\right|<2^{2 n+1} c_{n} \epsilon_{1}|z|^{n}, \quad|z| \leqq \epsilon_{2} .
$$

Also

$$
\left|i \cdot Z^{(n)}(z)-i z^{n}\right|<2^{2 n+1} c_{n} \epsilon_{1}|z|^{n}, \quad|z| \leqq \epsilon_{2},
$$

so that for $a=\alpha+i \beta \neq 0,|z| \leqq \epsilon_{2}$,

$$
\left|a \cdot Z^{(n)}(z)-a z^{n}\right|<2^{2(n+1)} c_{n} \epsilon_{1}|a||z|^{n}
$$

and

$$
\left|a \cdot Z^{(n)}(z)\right|>|a||z|^{n}\left(1-2^{2(n+1)} c_{n} \epsilon_{1}\right) .
$$

Choosing $\epsilon_{1}$ so small that

$$
\delta=\left\{a \mid\left(1-2^{2(n+1)} c_{n} \epsilon_{1}\right)>0\right.
$$

and setting $\epsilon_{2}\left(\epsilon_{1}\right)=\epsilon$, we get

$$
\left|a \cdot Z^{(n)}(z)\right|>\delta|z|^{n}, \quad \text { for }|z| \leqq \epsilon .
$$

We consider next case (II). Plainly

$$
g(z)=\sigma_{0} \Re\left\{a \cdot Z^{(n)}(z)\right\}+i \tau_{0} \Im\left\{a \cdot Z^{(n)}(z)\right\}
$$

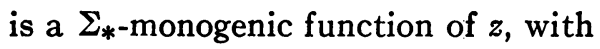

$$
\Sigma_{*}=\left\|\begin{array}{ll}
\sigma_{1}(x) / \sigma_{0} & \tau_{1}(y) / \tau_{0} \\
\sigma_{2}(x) / \sigma_{0} & \tau_{2}(y) / \tau_{0}
\end{array}\right\|
$$

A simple calculation shows that the first $(n-1) \Sigma_{*-}$ and $\Sigma_{*}^{\prime}$-derivatives of $g$ vanish identically, whereas the $n$th derivative is a nonvanishing constant, say $a_{*}$. Since $g(0)=0$ we have

$$
g(z)=a_{*} \cdot Z_{*}^{(n)}(z),
$$

$a_{*} \cdot Z_{*}^{(n)}$ being the formal power obtained from $\Sigma_{*}$ in the same way as $a \cdot Z^{(n)}$ was obtained from $\Sigma$. Obviously

$$
\left|a \cdot Z^{(n)}(z)\right| \geqq m_{\Sigma}^{-1}(0)|g(z)| .
$$

But $\Sigma_{*}$ satisfies condition (I). Thus case (II) is reduced to case (I).

Finally, the general case can be reduced to case (II). Let $\zeta=\xi+i \eta$ be a 
new independent variable defined by

$$
z=x+i y=\left(\sigma_{1}(0) \tau_{2}(0)\right)^{1 / 2} \xi+i\left(\sigma_{2}(0) \tau_{1}(0)\right)^{1 / 2} \eta .
$$

A simple calculation shows that a $\Sigma$-monogenic function $f(z)$ is a $\Sigma_{*-\text {-mono- }}$ genic function of $\zeta$ where

$$
\Sigma_{*}=\left\|\begin{array}{ll}
\left(\sigma_{2}(0) / \sigma_{1}(0)\right)^{1 / 2} \sigma_{1}\left[\left(\sigma_{1}(0) \tau_{2}(0)\right)^{1 / 2} \xi\right], & \left(\tau_{2}(0) / \tau_{1}(0)\right)^{1 / 2} \tau_{1}\left[\left(\sigma_{2}(0) \tau_{1}(0)\right)^{1 / 2} \eta\right] \\
\left(\sigma_{1}(0) / \sigma_{2}(0)\right)^{1 / 2} \sigma_{2}\left[\left(\sigma_{1}(0) \tau_{2}(0)\right)^{1 / 2} \xi\right], & \left(\tau_{1}(0) / \tau_{2}(0)\right)^{1 / 2} \tau_{2}\left[\left(\sigma_{2}(0) \tau_{1}(0)\right)^{1 / 2} \eta\right]
\end{array}\right\|
$$

and that

$$
d_{\Sigma} f / d_{\Sigma} z=\left(\sigma_{2}(0) \tau_{2}(0)\right)^{-1 / 2} d_{\Sigma *} f / d_{\Sigma_{*}} \zeta
$$

Similarly a $\Sigma^{\prime}$-monogenic function $h(z)$ is a $\Sigma_{*}^{\prime}$-monogenic function of $\zeta$ and

$$
d_{\Sigma^{\prime}} h / d_{\Sigma^{\prime}} z=\left(\sigma_{2}(0) \tau_{2}(0)\right)^{-1 / 2} d_{\Sigma_{*}} h / d_{\Sigma_{*}^{\prime}}^{\prime} \text {. }
$$

Thus the first $(n-1) \Sigma_{*}$ - and $\Sigma_{*}^{\prime}$-derivatives of $a \cdot Z^{(n)}(z)$ (regarded as a function of $\zeta$ ) vanish, whereas the $n$th equals $\left[\sigma_{2}(0) \tau_{2}(0)\right]^{n / 2} a$. Therefore

$$
a \cdot Z^{(n)}(z)=\left[\sigma_{2}(0) \tau_{2}(0)\right]^{n / 2} a \cdot Z_{*}^{(n)}(\zeta),
$$

$Z_{*}^{(n)}$ again denoting the formal power formed with respect to $\Sigma_{*}$. Since

$$
|\zeta| \geqq m_{\Sigma}^{-1}(0)|z|
$$

and since $\Sigma_{*}$ satisfies condition (II), the proof is complete.

Lemma 5.3. Let $\Sigma$ be a normal matrix. Given a positive integer $n$ and two constant $z_{0}$ and $a \neq 0$, there exist two positive constants $\delta$ and $R$ (both depending upon $\Sigma$ ) such that

$$
\left|a \cdot Z^{(n)}\left(z_{0}, z\right)\right|>\delta\left|z-z_{0}\right|^{n}, \quad \text { for }\left|z-z_{0}\right| \geqq R \text {. }
$$

Proof. Again we may assume that $z_{0}=0$. Consider the matrices

$$
\begin{array}{ll}
\Sigma_{1}=\left\|\begin{array}{cc}
\sigma_{1}(|x|) & \tau_{1}(|y|) \\
\sigma_{2}(|x|) & \tau_{2}(|y|)
\end{array}\right\|, & \Sigma_{2}=\left\|\begin{array}{cc}
\sigma_{1}(-|x|) & \tau_{1}(|y|) \\
\sigma_{2}(-|x|) & \tau_{2}(|y|)
\end{array}\right\|, \\
\Sigma_{3}=\left\|\begin{array}{cc}
\sigma_{1}(-|x|) & \tau_{1}(-|y|) \\
\sigma_{2}(-|x|) & \tau_{2}(-|y|)
\end{array}\right\|, \quad \Sigma_{4}=\left\|\begin{array}{cc}
\sigma_{1}(|x|) & \tau_{1}(-|y|) \\
\sigma_{2}(|x|) & \tau_{2}(-|y|)
\end{array}\right\|
\end{array}
$$

and denote the formal powers formed with respect to $\Sigma_{j}$ by $a \cdot Z_{j}^{(n)}$. Obviously, for $z=r e^{i \theta}, 0 \leqq \theta \leqq 2 \pi$,

$$
a \cdot Z^{(n)}(z)=a \cdot Z_{j}^{(n)}(z), \quad \text { for }(j-1) \pi / 2 \leqq \theta \leqq j \pi / 2, j=1,2,3,4 .
$$

Therefore it will be sufficient to prove our lemma for the case when

$$
\sigma_{j}(+\infty)=\sigma_{j}(-\infty), \quad \tau_{j}(+\infty)=\tau_{j}(-\infty), \quad j=1,2 .
$$


This case can be reduced (in the same way as in the proof of Lemma 5.2) to the case

$$
\sigma_{j}(+\infty)=\sigma_{j}(-\infty)=\tau_{j}(+\infty)=\tau_{j}(-\infty)=1, \quad j=1,2 .
$$

We assume (5.6) and say that for any positive integer $n$

$$
\left|X^{(n)}-x^{n}\right|=o\left(|x|^{n}\right), \quad|x| \rightarrow \infty
$$

(and similarly for $X^{*(n)}, Y^{(n)}, Y^{*(n)}$ ). In fact, by (5.6) and l'Hospital's rule

$$
\int_{0}^{x}\left|1-1 / \sigma_{1}\right| d x=o(|x|), \quad \int_{0}^{x}\left|1-\sigma_{2}\right| d x=o(|x|)
$$

and therefore

$$
\left|X^{(1)}(x)-x\right|=\left|\int_{0}^{x}\left(1 / \sigma_{2}-1\right) d x\right|=o(|x|) .
$$

Thus (5.7) holds for $n=1$. If it holds for $n-1$, we have, say for $x>0$ and an even $n$,

$$
\begin{aligned}
\left|X^{(n)}(x)-x^{n}\right| & =n\left|\int_{0}^{x}\left(\sigma_{2} X^{(n-1)}-x^{n-1}\right) d x\right| \\
& \leqq n\left|\int_{0}^{x} \sigma_{2}\left(X^{(n-1)}-x^{n-1}\right) d x\right|+n\left|\int_{0}^{x} x^{n-1}\left(\sigma_{2}-1\right) d x\right| \\
& <n m_{\Sigma}(\infty) \int_{0}^{x} o\left(|x|^{n-1}\right) d x+n|x|^{n-1} o(|x|)=o\left(|x|^{n}\right) .
\end{aligned}
$$

(Note that for a normal matrix $m_{\Sigma}(\infty)<\infty$.)

Now

$$
\begin{aligned}
\left|Z^{(n)}(z)-z^{n}\right|= & \left|\sum_{\nu=0}^{n} C_{n, \nu} i^{n-\nu}\left[X^{(\nu)}(x) Y^{(n-\nu)}(y)-x^{\nu} y^{n-\nu}\right]\right| \\
\leqq & \sum_{\nu=1}^{n} C_{n, \nu}\left|X^{(\nu)}(x)-x^{\nu}\right|\left|Y^{(n-\nu)}(y)\right| \\
& +\sum_{\nu=0}^{n-1} C_{n, \nu}\left|Y^{(n-\nu)}(y)-y^{n-\nu}\right||x|^{\nu} \\
= & \sum_{\nu=1}^{n} C_{n, \nu} O\left(|z|^{\nu}\right) O\left(|z|^{n-\nu}\right) \\
& +\sum_{\nu=0}^{n-1} C_{n, \nu} o\left(|z|^{n-\nu}\right) O\left(|z|^{\nu}\right)=o\left(|z|^{n}\right),
\end{aligned}
$$

and similarly

$$
\left|i \cdot Z^{(n)}(z)-i z^{n}\right|=o\left(|z|^{n}\right)
$$


whence it follows easily that for any constant $a$

$$
\left|a \cdot Z^{(n)}(z)-a z^{n}\right|=o\left(|z|^{n}\right)
$$

so that

$$
\left|a \cdot Z^{(n)}(z)\right|>|a||z|^{n}-o\left(|z|^{n}\right)
$$

and therefore

$$
\left|a \cdot Z^{(n)}(z) / z^{n}\right| \geqq|a|-o(1), \quad|z| \rightarrow \infty,
$$

so that for suitably chosen $\delta>0$ and $R>0$

$$
\left|a \cdot Z^{(n)}(z)\right|>\delta|z|^{n}, \quad|z| \geqq R .
$$

Thus the lemma is proved.

Finally we consider the matrix $\Sigma(\lambda)$ (defined by (1.7)) and denote by $a \cdot Z_{\lambda}^{(n)}(z)$ the formal powers constructed with respect to this matrix. Re-examining the proof of Lemma 5.3 and recalling (1.9) as well as the obvious fact that

$$
\left|1-\alpha^{\lambda}\right|<|1-\alpha|, \quad \alpha>0, \quad 0 \leqq \lambda \leqq 1,
$$

we see that the following somewhat stronger lemma is true.

Lemma 5.4. Let $\Sigma$ be a normal matrix. Given a positive integer $n$ and two constants $a \neq 0$ and $z_{0}$ there exist two positive constants $R$ and $\delta$ such that

$$
\left|a \cdot Z_{\lambda}^{(n)}\left(z_{0} ; z\right)\right|>\delta\left|z-z_{0}\right|^{n}, \quad \text { for } \quad\left|z-z_{0}\right| \geqq R, \quad 0 \leqq \lambda \leqq 1 .
$$

In proving the above lemma we made use only of hypothesis $\beta^{\prime}(\operatorname{not} \beta)$.

6. $\Sigma$-derivatives of higher order. We define higher $\Sigma$-derivatives of a $\Sigma$-monogenic function $f(z)$ by

$$
\begin{aligned}
& f^{[0]}(z)=f(z), \\
& f^{[n]}(z)= \begin{cases}d_{\Sigma} f^{[n-1]}(z) / d_{\Sigma z}, & \text { if } n \text { is odd, } \\
d_{\Sigma^{\prime}} f^{[n-1]}(z) / d_{\Sigma} z, & \text { if } n \text { is even. }\end{cases}
\end{aligned}
$$

Plainly $f^{[2 n]}$ is $\Sigma$-monogenic and $f^{[2 n+1]} \Sigma^{\prime}$-monogenic.

If we write

$$
f^{[n]}(z)=u^{[n]}+i v^{[n]}
$$

and set

$$
D_{1} \phi=\sigma_{1} \partial \phi / \partial x, \quad D_{2} \phi=\left(\sigma_{2}\right)^{-1} \partial \phi / \partial x,
$$

we have by (2.3) and (1.5)

$$
\begin{aligned}
u^{[2 n+1]} & =D_{1}\left(D_{2} D_{1}\right)^{n} u, & v^{[2 n+1]} & =D_{2}\left(D_{1} D_{2}\right)^{n} v, \\
u^{[2 n]} & =\left(D_{2} D_{1}\right)^{n} u, & v^{[2 n]} & =\left(D_{1} D_{2}\right)^{n} v .
\end{aligned}
$$

LEMMA 6.1. Let $F(z), G(z), H(z)$ be analytic functions of $z$ defined in the 
neighborhood of the origin. Set

$$
\begin{aligned}
& F_{0}(z)=F(z), \\
& F_{n}(z)= \begin{cases}G(z) F_{n-1}^{\prime}(z), & \text { if } n \text { is odd, } \\
H(z) F_{n-1}^{\prime}(z), & \text { if } n \text { is even. }\end{cases}
\end{aligned}
$$

Then there exists a positive constant $C$ such that

$$
\left|F_{n}(0)\right| \leqq n ! C^{n}
$$

Proof $\left({ }^{(}\right)$. Set

$$
F(z)=\sum \alpha_{n} z^{n}, \quad G(z)=\sum \beta_{n} z^{n}, \quad H(z)=\sum \gamma_{n} z^{n}
$$

and consider three other analytic functions

$$
F^{*}(z)=\sum \alpha_{n}^{*} z^{n}, \quad G^{*}(z)=\sum \beta_{n}^{*} z^{n}, \quad H^{*}(z)=\sum \gamma_{n}^{*} z^{n}
$$

such that

$$
0 \leqq\left|\alpha_{n}\right| \leqq \alpha_{n}^{*}, \quad 0 \leqq\left|\beta_{n}\right| \leqq \beta_{n}^{*}, \quad 0 \leqq\left|\gamma_{n}\right| \leqq \gamma_{n}^{*} .
$$

Let $F_{n}^{*}(z)$ be defined in the same way as $F_{n}(z)$. Since $F_{n}(0)$ is a linear combination of the coefficients $\alpha_{\nu}, \beta_{\mu}, \gamma_{\lambda}$ with positive coefficients, we have

$$
\left|F_{n}(0)\right| \leqq F_{n}^{*}(0) \text {. }
$$

Let $M>0$ be so large and $\delta>0$ be so small that for $|z| \leqq \delta, F, G, H$ are regular and $|F|,|G|,|H|$ are not greater than $M$. By Cauchy's inequality

$$
\left|\alpha_{n}\right|,\left|\beta_{n}\right|,\left|\gamma_{n}\right| \leqq M \delta^{-n} \text {. }
$$

Therefore we may set

Then

$$
F^{*}=G^{*}=H^{*}=M \delta /(\delta-z) .
$$

so that

$$
F_{n}^{*}(0)=1 \cdot 3 \cdots \cdot(2 n-1) M^{n+1} / \delta^{n} .
$$

(6.6) and (6.5) imply (6.4).

Lemma 6.2. If $f(z)$ is $\Sigma$-monogenic at $z=z_{0}$, then there exists a constant $C$ such that

$$
\left|f^{[n]}\left(z_{0}\right)\right|<n ! C^{n} .
$$

Proof. Without loss of generality we set $z_{0}=0$. Noting (6.3) and applying the previous lemma to the case

(9) The original proof has been simplified due to a suggestion made by S. Bergman. 


$$
F(z)=u(z, 0), \quad G(z)=\sigma_{1}(z), \quad H(z)=1 / \sigma_{2}(z),
$$

we see that

$$
\left|u^{[n]}(0,0)\right|<n ! C_{1}^{n}
$$

Similarly

$$
\left|v^{[n]}(0,0)\right|<n ! C_{2}^{n}
$$

so that

$$
\left|f^{[n]}(0)\right|<n !\left(C_{1}+C_{2}\right)^{n} .
$$

TheOREM 6.1. If $f(z)$ is $\Sigma$-monogenic at $z=z_{0}$ and if

$$
f^{[n]}\left(z_{0}\right)=0, \quad n=0,1, \cdots,
$$

then

$$
f \equiv 0 \text {. }
$$

Proof. Without loss of generality we set $z_{0}=0$. It follows from (6.3) that

$$
u^{[n]}=\phi_{n}+\psi_{n} \partial^{n} u / \partial x^{n},
$$

where $\phi_{n}$ is a linear combination of products of partial derivatives of $u$ with respect to $x$ of order less than $n$ and $\psi_{n}$ is a nonvanishing function. Hence (6.8) implies that

$$
\left(\partial^{n} u / \partial x^{n}\right)_{x=y=0}=0,
$$$$
n=0,1, \cdots
$$

Since $u(x, 0)$ is an analytic function of $x$

$$
u(x, 0) \equiv 0 \text {. }
$$

Similarly

$$
v(x, 0) \equiv 0
$$

and therefore

$$
(\partial v / \partial x)_{y=0}=d v(x, 0) / d x \equiv 0
$$

Thus

$$
(\partial u / \partial y)_{y=0}=-\left(\tau_{2}(0) / \sigma_{2}(x)\right)(\partial v / \partial x)_{y=0} \equiv 0 .
$$

But $u$ is a solution of a partial differential equation of second order with analytic coefficients (1.3a). Since for $y=0, u=0, \partial u / \partial y=0$, it follows from the Cauchy-Kowalewski theorem that $u \equiv 0$. Hence $v$ is constant and so is $f$. But $f(0)=0$ so that $f$ vanishes identically.

7. Formal power series. By virtue of Theorem 3.7, a formal power series

$$
\sum_{n=0}^{\infty} a_{n} \cdot Z^{(n)}(a ; z)
$$


represents a $\Sigma$-monogenic function in any domain in which it converges uniformly. We shall postpone the discussion of the exact domain of convergence of (7.1) and prove only

\section{THEOREM 7.1. If the ordinary power series}

$$
\sum_{n=0}^{\infty} a_{n} z^{n}
$$

possesses a nonvanishing radius of convergence, then there exists a neighborhood $D$ of $z$ such that (7.1), as well as all series obtained by $\Sigma$ - and $\Sigma^{\prime}$-differentiating (7.1) term by term, converges uniformly and absolutely in $D$.

Proof. Let $D$ be the domain given by

$$
|z-a| \leqq \rho / 2^{1 / 2} m_{\Sigma}\left(\left|z_{0}\right|+r\right),
$$

$$
\rho<r
$$

where $r$ is the radius of convergence of (7.2); then $D$ possesses the required property. For, in $D,(7.1)$ satisfies the inequality (cf. (5.2))

$$
\sum_{n=0}^{N}\left|a_{n} \cdot Z^{(n)}(a ; z)\right| \leqq 2 \sum_{n=0}^{N}\left|a_{n}\right| \rho^{n},
$$

and a similar argument holds for the derived series.

Thus formal power series may be $\Sigma$ - and $\Sigma^{\prime}$-differentiated term by term. Therefore, if

$$
f(z)=\sum_{n=0}^{\infty} a_{n} \cdot Z^{(n)}(a ; z)
$$

we have

$$
n ! a_{n}=f^{[n]}(a) .
$$

Now let $f(z)$ be an arbitrary $\Sigma$-monogenic function defined at $z=a$. We de? termine the coefficients $a_{n}$ from (7.4) and use these coefficients to form the series (7.2). By virtue of Theorem 6.1 this series possesses a nonvanishing radius of convergence. With the same coefficients we form the series (7.1). By virtue of Theorem 7.1 this series converges uniformly and absolutely in some neighborhood of $a$. The function it represents possesses at $a$ the $\left(\Sigma, \Sigma^{\prime}\right)$ derivatives $n ! a_{n}$. Therefore, by Theorem 6.1 , the series represents $f(z)$. Thus we have proved

THEOREM 7.2. A function which is $\Sigma$-monogenic at $z=a$ can be (uniquely) expanded in a formal power series of the form (7.1).

We next establish

THEOREM 7.3. $\Sigma$-monogenic functions possess zeros of only a finite integral order. 
This means: If $f(z)$ is $\Sigma$-monogenic at $z=a$ and if $f(a)=0$, then there exists an integer $k>0$ such that

$$
1 / C<\left|f(z) /(z-a)^{k}\right|<C, \text { for }|z-a|<\epsilon,
$$

where $C$ and $\epsilon$ are appropriately chosen positive constants.

Corollary. All zeros of a $\Sigma$-monogenic function are isolated points. A $\Sigma$-monogenic function is uniquely determined by its values at any infinite sequence of points, provided it is regular at a limit point of the sequence.

Proof. By Theorem 7.2, $f(z)$ has the form

$$
f(z)=\sum_{n=k}^{\infty} a_{n} \cdot Z^{(n)}(a ; z),
$$

By Lemma 5.1 and Lemma 5.2 there exist two positive constants $\delta$ and $\epsilon$ such that for $|z-a| \leqq \epsilon$

$$
|f(z)|>\delta|z-a|^{k}-2 \sum_{n=k+1}^{\infty}\left|a_{n}\right|\left\{2^{1 / 2} m_{\Sigma}(|a|+\epsilon)|z-a|\right\}^{n},
$$

and

$$
|f(z)| \leqq 2 \sum_{n=k}^{\infty}\left|a_{n}\right|\left\{2^{1 / 2} m_{\Sigma}(|a|+\epsilon)|z-a|\right\}^{n}
$$

Therefore

$$
|f(z)| /|z-a|^{k} \geqq \delta+o(|z-a|),
$$

and

$$
\frac{|f(z)|}{|z-a|^{k}} \leqq 2\left(2^{k}\right)^{1 / 2} m_{\Sigma}^{k}(|a|+\epsilon)+o(|z-a|), \quad z \rightarrow a,
$$

which proves the theorem.

We see that $a$ is a zero of the $k$ th order if and only if

$$
f^{[0]}(a)=f^{[1]}(a)=\cdots=f^{[k-1]}(a)=0, f^{[k]}(a) \neq 0 .
$$

If we replace hypothesis $\beta$ by $\beta^{\prime}$ the proof of Theorem 7.1 remains the same, whereas that of Theorem 7.3 breaks down. Theorem 7.3 is still true, but only for functions for which the existence of a formal Taylor development at $z=a$ is known a priori.

8. Correspondence between $\Sigma$-monogenic and analytic functions. Let $f(z)$ be $\Sigma$-monogenic at $z=a$ and $\phi(z)$ analytic at this point. We say that at $a f$ corresponds to $\phi$ if

$$
f^{[n]}(a)=\phi^{(n)}(a), \quad n=0,1, \ldots .
$$

By the results of the preceding section the existence of $f$ implies the existence 
of $\phi$ and vice versa $\left({ }^{10}\right)$. (This concerns the existence of the functions in the neighborhood of $a$. )

Instead of (8.1) we write

$$
f \sim \phi
$$

(at $a$ ). If (at $a)$

$$
f \sim \phi \text { and } g \sim \psi,
$$

where $f$ and $g$ are $\Sigma$-monogenic and $\phi$ and $\psi$ are analytic functions, then (at this point)

and

$$
\begin{array}{rlr}
f+g & \sim \phi+\psi, \\
\alpha f & \sim \alpha \phi \\
f^{[n]} & \sim \phi^{(n)}, & \quad(\alpha \text { a real constant }),
\end{array}
$$

$$
F \sim \Phi
$$

where

$$
F(z)=\int_{a}^{z} f d_{\Sigma} z, \quad \Phi(z)=\int_{a}^{z} \phi d z .
$$

If $f(z)$ is $\Sigma$-monogenic, $f_{1}(z) \Sigma_{1}$-monogenic, and $\phi(z)$ analytic and if $f \sim \phi$, $f_{1} \sim \phi$ (at $a$ ), we write $f \sim f_{1}$ (at $a$ ).

If at $a$

$$
f \sim \phi, \quad g \sim \psi,
$$

then the function $h$ which corresponds to the analytic function $\chi=\phi \psi$ (at $a$ ) shall be called the formal product of $f$ and $g, a$ being the center of multiplication. For this product we write

$$
h=f \cdot g \text {. }
$$

The usual laws can be extended to this formal multiplication, with one exception. If (8.4) holds, then

$$
f(b)=0, \quad b \neq a,
$$

does not necessarily imply that $h(b)=0$. Note that the function

$$
H=f(b) \cdot g \quad \text { (center of multiplication at } a \neq b \text { ) }
$$

(that is, the formal product of the constant $f(b)$ and the function $g$ ) is different from $h$. In general $H(b) \neq h(b)$.

THEOREM 8.1. If $\phi(z)$ is an entire analytic function and if

$$
f \sim \phi \quad(\text { at } a)
$$

( $f$ being $\Sigma$-monogenic), then the formal power series (around a) representing $f$

(10) The concept of corresponding functions is a useful one in applied problems (cf. [4]). 
converges uniformly and absolutely in every finite domain. For every point $b \neq a$ there exists an entire analytic function $\psi(z)$ such that

$$
f \sim \psi \quad(\text { at } b) .
$$

Proof. The series

$$
\phi(z)=\sum_{n=0}^{\infty} a_{n}(z-a)^{n}
$$

converges in the whole finite plane. By the argument used in proving Theorem 7.1 this fact implies that the series

$$
\sum_{n=0}^{\infty} a_{n} \cdot Z^{(n)}(z)=f(z)
$$

converges uniformly and absolutely for $|z| \leqq R<\infty$. Set

$$
\Phi(z)=2 \sum_{n=0}^{\infty}\left[m_{\Sigma}(|a|+|b|) 2^{1 / 2}\right]^{n}\left|a_{n}\right| z^{n}
$$

$\Phi$ is an entire function. By (8.6) and Lemma 5.1

Therefore

$$
\left|f^{[n]}(b)\right|<\Phi^{(n)}(|b-a|) \text {. }
$$

$$
\psi(z)=\sum_{n=0}^{\infty} \frac{f^{[n]}(b)}{n !} z^{n}
$$

is an entire function satisfying (8.5).

9. Formal polynomials. We shall call a $\Sigma$-monogenic function a formal polynomial of the $n$th degree, if at some point $a$ it corresponds to a polynomial of the $n$th degree, that is, if

$$
f(z)=a_{0}+a_{1} \cdot Z^{(1)}(a ; z)+\cdots+a_{n} \cdot Z^{(n)}(a ; z), \quad a_{n} \neq 0 .
$$

TheOREM 9.1. A formal polynomial of the nth degree corresponds, at any point $b$, to a polynomial of the nth degree.

Proof. By Theorem 7.2, $a_{\nu} \cdot Z^{(\nu)}(a ; z)$ can be represented by a formal power series around $b$. Using (7.4) we obtain

$$
a_{\nu} \cdot Z^{(\nu)}(a ; z)=\sum_{\mu=0}^{\nu} C_{\nu, \mu}\left[a_{\nu} \cdot Z^{(\nu-\mu)}(a ; b)\right] \cdot Z^{(\mu)}(b ; z) .
$$

In this formula (a generalization of the binomial formula) the center of multiplication is at $b$ and $a_{\nu} \cdot Z^{(\nu-\mu)}(a ; b)$ is to be treated as a constant.

By virtue of (9.2), (9.1) can be written in the form

$$
f(z)=b_{0}+b_{1} \cdot Z^{(1)}(b ; z)+\cdots+b_{n} \cdot Z^{(n)}(b ; z) .
$$

It is easily seen that $a_{n} \neq 0$ implies $b_{n} \neq 0$. 
(9.2) could also be proved by direct computation. Therefore Theorem 9.1 holds independently of hypothesis $\beta$.

We now prove the following theorems.

TheOREM 9.2. If $\Sigma$ is a normal matrix, a formal polynomial of nth degree possesses exactly $n$ zeros (each zero being counted according to its multiplicity).

THEOREM 9.3. A formal polynomial of the nth degree possesses not more than $n$ zeros (each zero being counted according to its multiplicity).

We shall prove the first theorem without making use of hypothesis $\beta$. Therefore the second is implied by the first. For, let $f(z)$ be a formal polynomial of the $n$th degree,

$$
f(z)=\sum_{\nu=0}^{n} a_{\nu} \cdot Z^{(\nu)}(z),
$$$$
a \neq 0,
$$

possessing more than $n$ zeros. There exists a positive $A$ such that $f(z)$ possesses more than $n$ zeros within the square $|x|<A,|y|<A$. We define four functions $\sigma_{* j}(x), \tau_{* j}(y), j=1,2$, which need not satisfy hypothesis $(\beta)$, such that

for

$$
\sigma_{* j}(x)=\sigma_{j}(x), \quad \tau_{* j}(y)=\tau_{j}(y),
$$

$$
|x| \leqq A, \quad|y| \leqq A \text { and } \cdot j=1,2
$$

and such that

$$
\Sigma_{*}=\left\|\begin{array}{cc}
\sigma_{* 1} & \tau_{* 1} \\
\sigma_{* 2} & \tau_{* 2}
\end{array}\right\|
$$

is a normal matrix. Denoting by $a_{v} \cdot Z_{*}^{(\nu)}$ the formal powers obtained from the matrix $\Sigma_{*}$, we have

$$
a \cdot Z_{*}^{(v)}(z)=a \cdot Z^{(\nu)}(z) \quad \text { for } \quad|x| \leqq A, \quad|y| \leqq A .
$$

Therefore

$$
g(z)=\sum_{\nu=0}^{n} a_{\nu} \cdot Z_{*}^{(\nu)}(z)=f(z) \quad \text { for } \quad|x| \leqq A, \quad|y| \leqq A .
$$

Thus $g(z)$ possesses more than $n$ zeros. This contradicts Theorem 9.2.

Now for the proof of Theorem 9.2(11). Set

$$
f_{\lambda}(z)=\sum_{\nu=0}^{n} a_{\nu} \cdot Z_{\lambda}^{(\nu)}(z),
$$

the subscript $\lambda$ indicating that the formal powers have been obtained from the matrix $\Sigma(\lambda)$ (see $\S 1$ ). Using (1.9) and the Lemmas 5.1 and 5.4, we have for $0 \leqq \lambda \leqq 1$

(11) A formal polynomial can be decomposed into linear factors but this does not yield the existence of zeros. 


$$
\begin{aligned}
\left|f_{\lambda}(z)\right| & \geqq\left|a_{n} \cdot Z_{\lambda}^{(n)}(z)\right|-\sum_{\nu=0}^{n-1}\left|a_{\nu} \cdot Z_{\lambda}^{(\nu)}(z)\right| \\
& >\delta|z|^{n}-2 \sum_{\nu=0}^{n-1}\left|a_{\nu}\right| m_{\Sigma}^{\prime}(\infty)\left(2^{\nu}\right)^{1 / 2}|z|^{\nu} \quad \text { for } \quad|z| \geqq R,
\end{aligned}
$$

$\delta$ and $R$ being positive constants. Therefore there exist two positive constants $\alpha$ and $A$ such that

$$
\left|f_{\lambda}(z)\right| \geqq \alpha, \quad \text { for }|z| \geqq A, 0 \leqq \lambda \leqq 1 .
$$

Obviously $f_{\lambda}(z)$ is a continuous function of $\lambda$ (for a fixed $z$ ). By (9.3), $\log f_{\lambda}(z)$ is a continuous function of $\lambda$ (for $|z| \geqq A, 0 \leqq \lambda \leqq 1$ ) and so is

$$
I(\lambda)=(2 \pi i)^{-1} \oint_{|z|=A} d \log f_{\lambda}
$$

But $I(\lambda)$ is always an integer, therefore it is constant and $I(0)=I(1)$. Since

$$
f_{0}(z)=\sum_{\nu=0}^{n} a_{\nu} z^{\nu}
$$

$f_{0}(z)$ possess $n$ zeros. By (9.3) these zeros lie within $|z|=A$. Therefore $I(0)=n$ and

$$
I(1)=(2 \pi i)^{-1} \oint_{|z|=A} d \log f=n .
$$

(9.4) indicates that $f(z)$ possesses zeros within $z=A$ ( $A$ is so chosen that there are no zeros outside of this circle). Their number is finite, by virtue of Theorem 7.3. We denote these zeros by $z_{1}, z_{2}, \cdots, z_{N}$, and their respective multiplicities by $\nu_{1}, \nu_{2}, \cdots, \nu_{N}$. Around each $z_{j}$ we draw a circle $\left|z-z_{j}\right|=\epsilon_{j}$ such that all circles are situated within $|z|=A$ and no two circles intersect or include one another. Furthermore, we choose each $\epsilon_{j}$ so small that

$$
\left|f(z) /\left(z-z_{j}\right)^{v_{i}}\right|>0 \text { for }\left|z-z_{j}\right| \leqq \epsilon_{j} .
$$

This is possible by virtue of Theorem 7.3. Then

and therefore

$$
\oint_{\left|z-z_{j}\right|=\epsilon_{j}} d \log \frac{f(z)}{\left(z-z_{j}\right)^{v_{j}}}=0,
$$

$$
\oint_{\left|z-z_{j}\right|-\epsilon_{j}} d \log f(z)=\oint_{\left|z-z_{j}\right|=\epsilon_{j}} d \log \left(z-z_{j}\right)^{v_{j}}=2 \pi i v_{j} .
$$

By a familiar argument 


$$
\sum_{j=1}^{N} \oint_{\left|z-z_{j}\right|=\epsilon_{j}} d \log f=\oint_{|z|=A} d \log f .
$$

From (9.4), (9.5) and (9.6) we have

This proves our theorem.

$$
\sum_{j=1}^{N} \nu_{j}=n
$$

We are now in a position to prove a theorem in polynomial interpolation.

THEOREM 9.4. Given a constant $a \neq 0, N$ distinct constants $z_{1}, z_{2}, \cdots, z_{N}$, and $N$ sequences of constants $c_{j 0}, c_{j 1}, \cdots, c_{j p_{j}}, j=1,2, \cdots, N$, there exists one and only one formal polynomial

$$
f(z)=\sum_{k=0}^{n-1} a_{k} \cdot Z^{(k)}(z)+a \cdot Z^{(n)}(z), \quad n=\nu_{1}+\cdots+\nu_{N}+N,
$$

such that

$$
f^{[l]}\left(z_{j}\right)=c_{j l}, \quad l=0,1, \cdots, \nu_{j}, j=1,2, \cdots, N .
$$

Proof. (9.7) can be written in the form

$$
\begin{aligned}
& \Re g^{[l]}\left(z_{j}\right)=\Re\left[c_{j l}-A_{n l j}\right], \\
& \Im^{[l]}\left(z_{j}\right)=\Im\left[c_{j l}-A_{n l j}\right], \quad l=0,1, \cdots, \nu_{j}, j=1,2, \cdots, N,
\end{aligned}
$$

where

and

$$
g(z)=\sum_{k=0}^{n-1} a_{k} \cdot Z^{(k)}(z)
$$

We write

$$
A_{n l j}= \begin{cases}\frac{n !}{(n-l) !} a \cdot Z^{(n-l)}\left(z_{j}\right), & \text { if } n-l \text { is even, } \\ \frac{n !}{(n-l) !} a \cdot \tilde{Z}^{(n-l)}\left(z_{j}\right), & \text { if } n-l \text { is odd. }\end{cases}
$$

$$
a_{k}=\alpha_{k}+i \beta_{k} .
$$

$\Re g^{[l]}\left(z_{j}\right)$ and $\Im^{[l]}\left(z_{j}\right)$ are linear combinations of $\alpha_{0}, \cdots, \alpha_{n-1}, \beta_{0}, \cdots, \beta_{n-1}$. Therefore (9.8) is a nonhomogeneous system of $2\left(\nu_{1}+\cdots+\nu_{N}+N\right)=2 n$ equations for the $2 n$ unknowns $\alpha_{0}, \cdots, \beta_{n-1}$. Suppose that the corresponding homogeneous system possesses a non-trivial solution $A_{0}, \cdots, B_{n-1}$. Since the coefficients of the equations are real we may assume that $A_{0}, \cdots, B_{n-1}$ are real. Set

We have

$$
G(z)=\sum_{k=0}^{n-1}\left(A_{k}+i B_{k}\right) \cdot Z^{(k)}(z)
$$




$$
G^{[l]}\left(z_{j}\right)=0, \quad l=0, \cdots, \nu_{j} ; j=1, \cdots, N .
$$

Thus the formal polynomial, $G(z)$, of a degree less then $n$ possesses $\nu_{1}+\ldots$ $+\nu_{N}+N=n$ zeros. This is impossible by virtue of Theorem 9.3. Therefore (9.8) always possesses a unique (real) solution and Theorem 9.4 is proved.

It follows from Theorem 9.4 that there exists a uniquely determined formal polynomial $f(z)$ of the $n$th degree, which possesses prescribed zeros $z_{1}, \cdots, z_{N}$ of prescribed multiplicity $\nu_{1}, \cdots, \nu_{N}, \nu_{1}+\cdots+\nu_{N}=n$, and a prescribed coefficient of highest order. (In the same way we could prescribe the lowest nonvanishing coefficient.)

Note that the coefficients of two formal polynomials of the same degree possessing the same zeros are in general not proportional.

10. $\Sigma$-exponential and $\Sigma$-trigonometric functions. In this section we consider $\Sigma$-monogenic functions which at a point $z=z_{0}$ correspond to the analytic functions

$$
a e^{\alpha\left(z-z_{0}\right)}, \quad a \sin \alpha\left(z-z_{0}\right), \quad a \cos \alpha\left(z-z_{0}\right) .
$$

These functions might be called $\Sigma$-exponential and $\Sigma$-trigonometric functions; they will be denoted by

$$
a \cdot E\left(z_{0} ; \alpha, z\right), \quad a \cdot S\left(z_{0} ; \alpha, z\right), \quad a \cdot C\left(z_{0} ; \alpha, z\right) .
$$

If $z_{0}=0$, we omit it. If $a=1$, we omit it.

THEOREM 10.1. If a $\Sigma$-monogenic function $f(z)$ corresponds to an analytic function of the form $a e^{\alpha z}+b \sin \alpha z+c \cos \alpha z$, at some point $z=z_{0}$, then $f(z)$ has the form

$$
a^{\prime} \cdot E(\alpha, z)+b^{\prime} \cdot S(\alpha, z)+c^{\prime} \cdot C(\alpha, z) .
$$

Proof. If we write down the formal power series for the functions (10.1) and apply (9.2) we obtain by a formal calculation the following "addition theorems":

(10.3) $a \cdot E\left(z_{0} ; \alpha, z\right)=\left\{a \cdot E\left(z_{0} ; \alpha, z_{1}\right)\right\} \cdot E\left(z_{1} ; \alpha, z\right)$,

(10.4) $a \cdot S\left(z_{0} ; \alpha, z\right)=\left\{a \cdot S\left(z_{0} ; \alpha, z_{1}\right)\right\} \cdot C\left(z_{1} ; \alpha, z\right)+\left\{a \cdot C\left(z_{0} ; \alpha, z_{1}\right)\right\} \cdot S\left(z_{1} ; \alpha, z\right)$,

$(10.5) a \cdot C\left(z_{0} ; \alpha, z\right)=\left\{a \cdot C\left(z_{0} ; \alpha, z_{1}\right)\right\} \cdot C\left(z_{1} ; \alpha, z\right)-\left\{a \cdot S\left(z_{0} ; \alpha, z_{1}\right)\right\} \cdot S\left(z_{1} ; \alpha, z\right)$.

In these formulae the expressions $\{\cdots\}$ are to be treated as constants.

Now, by hypothesis $f(z)$ has the form

$$
a_{1} \cdot E\left(z_{0} ; \alpha, z\right)+b_{1} \cdot S\left(z_{0} ; \alpha, z\right)+c_{1} \cdot C\left(z_{0} ; \alpha, z\right),
$$

where

$$
a_{1}=a e^{\alpha z_{0}}, \quad b_{1}=b \cos \alpha z_{0}-c \sin \alpha z_{0}, \quad c_{1}=b \sin \alpha z_{0}+c \cos \alpha z_{0} .
$$

From (10.3)-(10.5) it follows that $f(z)$ can be written in the form (10.2) where 


$$
\begin{gathered}
a^{\prime}=a_{1} \cdot E\left(z_{0} ; \alpha, 0\right), \quad b^{\prime}=b_{1} \cdot C\left(z_{0} ; \alpha, 0\right)-c_{1} \cdot S\left(z_{0} ; \alpha, 0\right), \\
c^{\prime}=b_{1} \cdot S\left(z_{0} ; \alpha, 0\right)+c_{1} \cdot C\left(z_{0} ; \alpha, 0\right) .
\end{gathered}
$$

By virtue of Theorem 10.1 it will suffice to consider the functions $E, S, C$. These functions possess many properties similar to those of the ordinary exponential and trigonometric functions. For instance, we have

$$
\begin{gathered}
E^{\prime \prime}-\alpha^{2} E=0, \quad S^{\prime \prime}+\alpha^{2} S=0, \quad C^{\prime \prime}+\alpha^{2} C=0, \\
S \cdot S+C \cdot C=1, \\
E(\alpha+\beta, z)=E(\alpha, z) \cdot E(\beta, z), \\
S(\alpha+\beta, z)=S(\alpha, z) \cdot C(\beta, z)+C(\alpha, z) \cdot S(\beta, z), \\
C(\alpha+\beta, z)=C(\alpha, z) \cdot C(\beta, z)-S(\alpha, z) \cdot S(\beta, z), \\
S(\alpha, z)+i \cdot C(\alpha, z)=E(i \alpha, z) .
\end{gathered}
$$

In these formulae the center of multiplication is at the origin.

Note that for real values of $\alpha, E(\alpha, z)$ is an entire function of $\alpha$. (The same applies to $S$ and $C$.) However, the valuie of this function for $\alpha=\alpha^{\prime}+i \alpha^{\prime \prime}$ is, in general, different from $E\left(\alpha^{\prime}+i \alpha^{\prime \prime}, z\right)$.

In what follows we consider only real values of $\alpha$. A simple formal calculation yields the separation of the functions $E, S, C$ into their real and imaginary parts. We set

$$
\begin{aligned}
& \operatorname{Sh}(\alpha, z)=\{E(\alpha, z)-E(-\alpha, z)\} / 2, \\
& \operatorname{Ch}(\alpha, z)=\{E(\alpha, z)+E(-\alpha, z)\} / 2 .
\end{aligned}
$$

(These functions correspond at the origin to the analytic functions $\sinh \alpha z$, $\cosh \alpha z$, respectively.) Then

$$
\begin{aligned}
& E(\alpha, z)=E(\alpha, x) \operatorname{Ch}(\alpha, i y)+\operatorname{Sh}(\alpha, i y), \\
& S(\alpha, z)=S(\alpha, x) C(\alpha, i y)+C(\alpha, x) S(\alpha, i y), \\
& C(\alpha, z)=C(\alpha, x) C(\alpha, i y)-S(\alpha, x) S(\alpha, i y) .
\end{aligned}
$$

Similar formulae hold for $i \cdot E, i \cdot S, i \cdot C$.

THEOREM 10.2. Essentially all(i2) particular solutions of the differential equations (10.3), (10.4) with the variables separated are obtained by forming the real and the imaginary parts of the functions

$$
E, i \cdot E, S, i \cdot S, C, i \cdot C, Z^{(1)}, i \cdot Z^{(1)}, Z^{(2)}, i \cdot Z^{(2)} \text {. }
$$

Proof. It will be sufficient to consider the differential equation for $u$. Setting

$$
u=\phi(x) \psi(y),
$$

(12) In a sense specified below. 
we obtain for $\phi$ and $\psi$ the ordinary differential equations

$$
\begin{aligned}
\left(\sigma_{1} \phi^{\prime}\right)^{\prime}+K \sigma_{2} \phi & =0, \\
\left(\psi^{\prime} / \tau_{2}\right)^{\prime}-K \psi / \tau_{1} & =0,
\end{aligned}
$$

$K$ being an arbitrary constant. For any choice of $K$ we obtain a system of 4 different particular solutions $u$,

$$
u=\phi_{i} \psi_{j},
$$$$
i, j=1,2 \text {, }
$$

where $\phi_{1}, \phi_{2}\left(\psi_{1}, \psi_{2}\right)$ are two linearly independent integrals of (10.9) (10.10).

It is easily seen that for $K>0$ such a system can be obtained by taking the real parts of the functions $S, C, i \cdot S, i \cdot C$, with $\alpha=K^{1 / 2}$. For $K<0$ we may take the real parts of $\mathrm{Sh}, \mathrm{Ch}, i \cdot \mathrm{Sh}, i \cdot \mathrm{Ch}$, with $\alpha=(-K)^{1 / 2}$. For $K=0$ we may set

$$
\begin{aligned}
& \phi_{1}=1, \quad \phi_{2}=\int_{0}^{x} d x / \sigma_{1}=X^{(1)}(x), \\
& \psi_{1}=1, \quad \psi_{2}=\int_{0}^{y} \tau_{2} d y=Y^{*(1)}(y) .
\end{aligned}
$$

Thus

$$
\begin{array}{ll}
\phi_{1} \psi_{1}=1, & \phi_{1} \psi_{2}=-\Re\left\{i \cdot Z^{(1)}(z)\right\}, \\
\phi_{2} \psi_{1}=\Re Z^{(1)}(z), & \phi_{2} \psi_{2}=-2^{-1} \Re\left\{i \cdot Z^{(2)}(z)\right\} .
\end{array}
$$

This completes the proof.

The similarity between the functions $E, S, C$ and the ordinary exponential and trigonometric functions is also exhibited by the following theorems (which could be extended easily and supplemented by others of the same kind).

TheOREM 10.3. The functions $E(\alpha, z)$ and $i \cdot E(\alpha, z)$ have no zeros.

Proof. Since $E(\alpha, z)=\Phi(x) \Psi(y)$ (cf. (10.6)), $E(\alpha, z)=0, z_{0}=x_{0}+i y_{0}$, would imply either $E(\alpha, z)=0$ for $\Re z=x_{0}$, or $E(\alpha, z)=0$ for $\Im_{z}=y_{0}$. Neither is possible by virtue of Theorem 7.3, since $E(\alpha, 0)=1$. The same argument holds for $i \cdot E$.

THEOREM 10.4. The functions $S(\alpha, z)$ and $C(\alpha, z), \alpha \neq 0$, have only real zeros.

Proof. Assume that

$$
S\left(\alpha, x_{0}+i y_{0}\right)=0,
$$

$y_{0} \neq 0$.

It is easily seen that

$$
C(\alpha, i y)=\sum_{n=0}^{\infty} \frac{\alpha^{2 n} Y^{(2 n)}(y)}{(2 n) !}, \quad S(\alpha, i y)=i \sum_{n=0}^{\infty} \frac{\alpha^{2 n+1} Y^{(2 n+1)}(y)}{(2 n+1) !} .
$$


Since $Y^{(2 n)}(y)$ and $y Y^{(2 n+1)}(y)$ are positive for $y \neq 0$,

$$
C\left(\alpha, i y_{0}\right) \neq 0, \quad S\left(\alpha, i y_{0}\right) \neq 0 .
$$

(10.7), (10.11) and (10.12) imply that $S\left(\alpha, x_{0}\right)=C\left(\alpha, x_{0}\right)=0$. Therefore $S(\alpha, z)=0$ for $\Re z=x_{0}$. This is impossible by virtue of Theorem 7.3. A similar argument holds for the function $C$.

11. Final remarks. In the hyperbolic case, $\sigma_{1} \sigma_{2} \tau_{1} \tau_{2}<0$. The formal part of our considerations can be carried out without any changes. Since the equations (1.3), (1.4) are now of hyperbolic type, the real and the imaginary parts of a $\Sigma$-monogenic function are not necessarily analytic functions of $x$ and $y$. Neither are the Lemmas 5.2 and 5.3 true. These indications may be sufficient.

If at $z=z_{0}, \sigma_{1} \sigma_{2} \tau_{1} \tau_{2}=0$, we call $z_{0}$ a critical point. A critical point lies on a critical line along which $\sigma_{1} \sigma_{2} \tau_{1} \tau_{2}=0$. The integrals defining the formal powers $Z(z), i \cdot Z(z)$ are not necessarily convergent when $z$ or $z_{0}$ are critical points, or when these points are separated by a critical line. If the integrals are convergent, they represent $\Sigma$-monogenic functions. In such a way it is possible to obtain particular solutions of partial differential equations which are elliptic in one part of the plane and hyperbolic in another. We also note that at a critical point the uniqueness Theorem 6.1 is not necessarily true.

Finally we note several open questions which seem to be of interest. What is the connection between the domains of existence of corresponding $\Sigma$-monogenic and analytic functions? Can a $\Sigma$-monogenic function, defined in a simply connected domain, be approximated by formal polynomials? Under what conditions is it possible to find a $\Sigma$-monogenic function which maps a given domain into a given domain?

\section{REFERENCES}

1. E. Beltrami, Sulle funzioni potenziali di sistemi simmetrici intorno ad un asse, Opere matematice, vol. 3, Milano, 1911, pp. 115-128.

2. - - Sulla teoria delle funzioni potenziali simmetriche, ibid. pp. 349-377.

3. S. Bergman, Linear operators in the theory of partial differential equations, Trans. Amer. Math. Soc. vol. 53 (1943) pp. 130-155.

4. L. Bers and A. Gelbart, On a class of differential equations in mechanics of continua, Quarterly of Applied Mathematics vol. 1 (1943) pp. 168-188.

5. E. Picard, Sur une système d'équations aux dérivêes partielles, C. R. Acad. Sci. Paris vol. 112 (1891) pp. 685-688.

6. - Sur une généralization des équations de la thêorie des fonctions d'une variable complexe, ibid. pp. 1399-1403.

7. S. Saks, Thérie de l'intégrale, Warsaw, 1932.

BROWN UNIVERSITY,

Providence, R. 1.

SYRACUSE UNIVERSITY,

SYRaCUSE, N. Y. 